Nutrition Science en évolution

La revue de l'Ordre professionnel des diététistes du Québec

\title{
Congrès annuel de l'American Society for Bone and Mineral Research
}

\section{Geneviève Mailhot}

Volume 17, numéro 1, printemps 2019

URI : https://id.erudit.org/iderudit/1062458ar

DOI : https://doi.org/10.7202/1062458ar

Aller au sommaire du numéro

Éditeur(s)

Ordre professionnel des diététistes du Québec

ISSN

2561-620X (numérique)

Découvrir la revue

Citer ce document

Mailhot, G. (2019). Congrès annuel de l'American Society for Bone and Mineral Research. Nutrition Science en évolution, 17(1), 39-41.

https://doi.org/10.7202/1062458ar d'utilisation que vous pouvez consulter en ligne. 


\section{ASBMR 2018 Annual Meeting}

September 28-October 1, 2018

Palais des Congrès de Montréal Montreal, Quebec, Canada

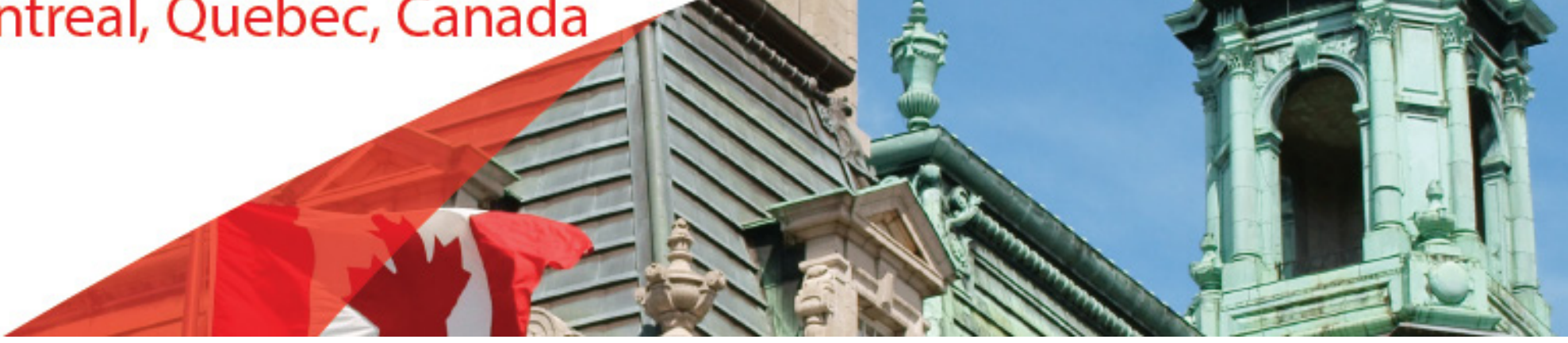

CONGRÈS ANNUEL
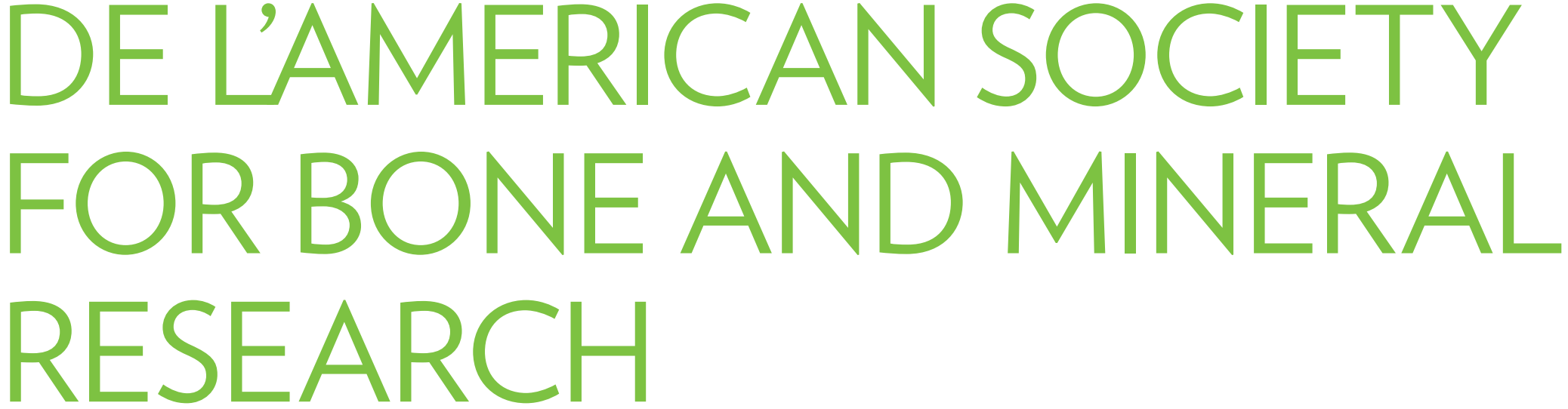

Geneviève Mailhot, Dt.P., Ph. D., professeure agrégée,

Département de nutrition, Université de Montréal

Le Congrès annuel de l'American Society for Bone and Mineral Research est le plus important rassemblement scientifique en santé osseuse. Il regroupe plus de 3000 participants provenant de plus de 70 pays. Cet événement s'est déroulé à Montréal du 28 septembre au $1^{\mathrm{er}}$ octobre 2018. Une sélection des dernières tendances en matière de nutrition et de santé osseuse est ici résumée. 


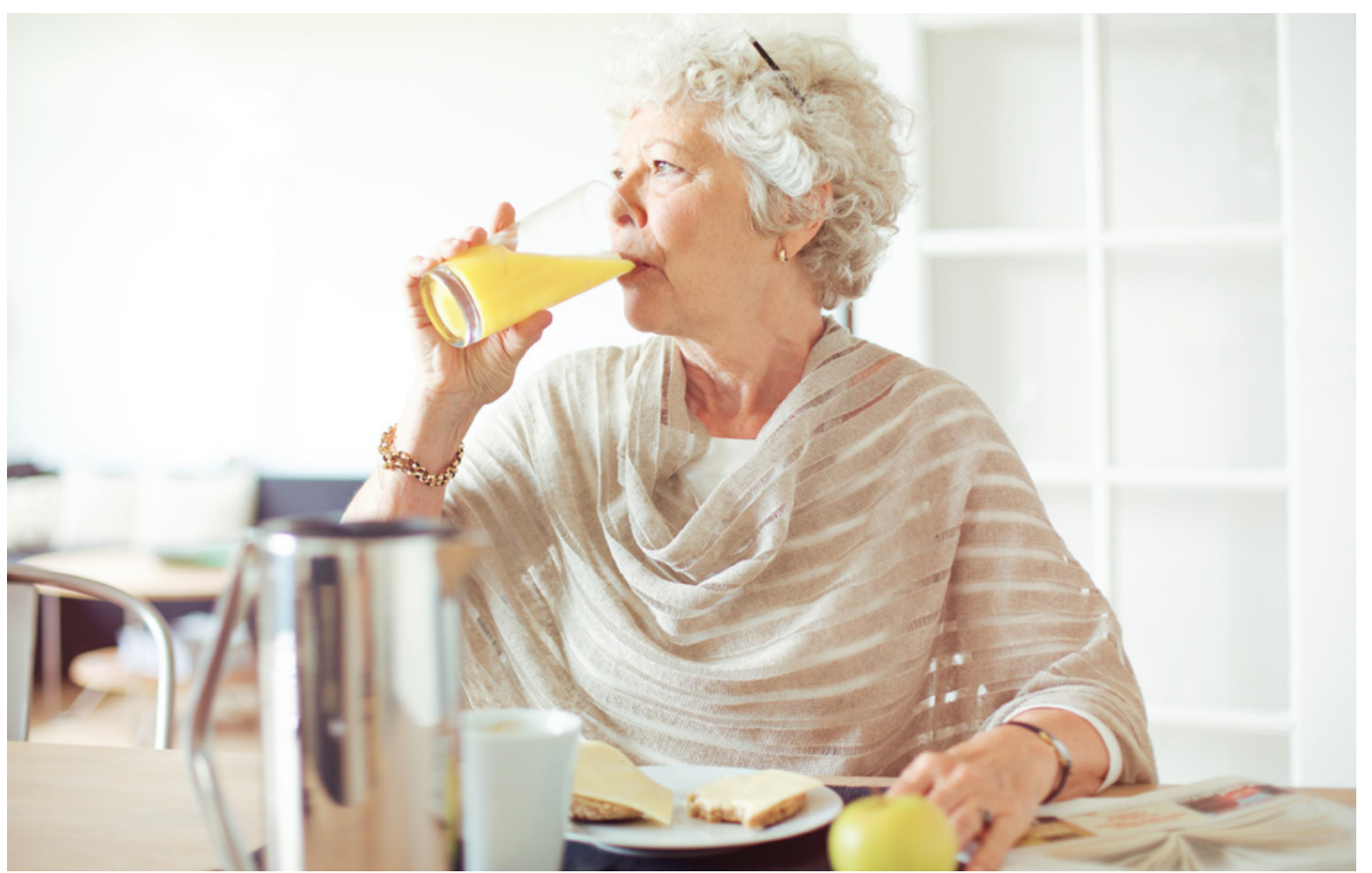

\section{OSTEOCALCIN FUNCTION ON ENERGY METABOLISM IS CONSERVED IN HUMANS: RESULTS OF A 5-YEAR PROSPECTIVE COHORT OF DIABETES ONSET}

\section{Cyrille Confavreux et} collaborateurs, INSERM UMR1033, Lyon, France

Des travaux réalisés chez la souris suggèrent que l'ostéocalcine, une protéine synthétisée par l'ostéoblaste, agit sur la cellule bêta du pancréas en modulant la sécrétion d'insuline. Or, l'association entre les niveaux sériques d'ostéocalcine et la survenue du diabète de type 2 n’avait jamais été étudiée de manière prospective chez les humains. Aussi, les auteurs ont mesuré les niveaux sériques d'ostéocalcine d'adultes prédiabétiques à leur entrée dans l'étude. Parmi les 323 participants suivis pendant 5 ans, 40 sujets ont reçu le diagnostic de diabète de type 2 durant cette période. Les auteurs ont montré une association inverse entre les niveaux sériques d'ostéocalcine et le risque de diabète de type 2 . Plus précisément, les personnes qui avaient une concentration sérique d'ostéocalcine dans le quar- tile inférieur présentaient un risque de diabète de type 2 presque triplé comparativement à celles ayant des concentrations d'ostéocalcine dans le quartile supérieur. Cette étude corrobore les travaux faits chez les rongeurs. Elle suggère que l'ostéocalcine régule également le métabolisme glucidique chez l'humain.

\section{BONE STRENGTH AND THE MICROBIOME}

\section{Christopher Hernandez,}

Cornell University, New York, États-Unis

Dr Hernandez a présenté des données précliniques fort intéressantes sur l'influence du microbiote sur la force osseuse et la possible implication de la vitamine K. L'administration d'antibiotiques dans l'eau de boisson des souris, dès lâage de 4 semaines jusqu’à 16 semaines, a modifié la composition et la diversité de leur microbiote. Bien que ces changements n'aient eu aucun effet sur la quantité osseuse (densité), la qualité osseuse (notée plus particulièrement par les propriétés du tissu minéral) elle, a été altérée. Une analyse plus fine des différences entre les groupes de souris a montré que des gènes associés à la synthèse de vita- mines étaient exprimés de manière différentielle entre les souris traitées aux antibiotiques et celles sans ce traitement. Parmi les différentes vitamines produites par le microbiote (biotine, vitamine $\mathrm{K}$, folates, riboflavine), une baisse de la concentration de vitamine $\mathrm{K}$ dans le cæcum des souris a été observée. Or, la vitamine K régule l'activité de certaines protéines de la matrice osseuse, telles l'ostéocalcine et la protéine gla de la matrice (matrix-gla-protein ou MGP en anglais). Dr Hernandez conclut en postulant que le microbiote exercerait un rôle non négligeable dans le contrôle de la qualité et de la force osseuse par l'entremise de la synthèse de la vitamine $\mathrm{K}$.

\section{THE CALGARY VITAMIN D STUDY: BONE MICROARCHITECTURE EFFECTS OF THREE-YEAR, SUPPLEMENTATION WITH 400, 4000 OR 10000 IU DAILY}

\author{
Lauren A. Burt, \\ University of Calgary, Calgary, \\ Canada
}

Les chercheurs de la Calgary Vitamin D Study ont étudié les effets de la vitamine D sur la santé osseuse. Des adultes de 55 à 70 ans $(\mathrm{n}=373)$ en santé, de la région de Calgary, ont reçu pendant 3 ans trois différentes doses de vitamine D3 (400, 4000 et 10000 UI). L'étude visait à déterminer si un apport en vitamine $D$ supérieur à l'AMT (4 000 UI) présentait des bienfaits additionnels pour la santé osseuse. Pour être incluses dans l'étude, ces personnes devaient avoir une santé osseuse optimale ou être ostéopéniques. Les personnes ostéoporotiques étaient exclues. Au début de l'étude, les sujets présentaient une concentration moyenne de 25 -hydroxyvitamine D de $78 \mathrm{nmol} / \mathrm{L}$. Au terme de l'étude, les individus ayant reçu 400, 4000 et 10000 UI de vitamine $D$ ont atteint des concentrations de 25-hydroxyvitamine D 
de 77, 132 et 144 nmol/L. La densité osseuse volumétrique au niveau du radius et du tibia a été mesurée par tomodensitométrie quantitative périphérique à haute résolution (HR-pQCT). Elle a révélé une perte de densité osseuse au tibia et au radius au cours des trois années chez les trois groupes sans changement toutefois de la force osseuse. De manière inespérée, cette perte s'est révélée plus marquée chez les personnes ayant reçu $10000 \mathrm{UI}$ de vitamine D. Les raisons de ce résultat demeurent toutefois inconnues. Il a été conclu que la prise d'une supplémentation en vitamine $\mathrm{D}$ à des doses supérieures à l'AMT pendant trois ans ne confère aucun avantage pour ralentir la perte osseuse associée au vieillissement. Finalement, bien qu'aucun effet indésirable sérieux n'ait été associé à la prise des trois doses de vitamine $\mathrm{D}$, les auteurs de cette étude ont rapporté une augmentation dose-dépendante des cas d'hypercalcémie et d'hypercalciurie.

\section{GLYCEMIC CONTROL INFLUENCES TRABECULAR MICROARCHITECTURE IN YOUTH WITH TYPE I DIABETES.}

\section{Deborah Mitchell,}

Massachusetts General Hospital, Boston, États-Unis

Le diabète affecte le remodelage osseux en diminuant la formation osseuse sans modifier la résorption osseuse. Il est bien documenté que les adultes ayant un diabète de type 1 présentent un risque de fractures plus élevé que des individus non diabétiques. Toutefois, cette relation demeure peu étudiée en pédiatrie. Cette étude regroupait 62 filles diabétiques de type 1 et 61 filles non diabétiques âgées en moyenne de 14 ans ne présentant aucune complication microvasculaire. Les participantes avaient le diabète depuis en moyenne 4,8 ans. La mesure de la densité osseuse du tibia et du radius par HR-pQCT a révélé que les filles diabétiques présentaient une diminution de la densité osseuse corticale et une augmentation de la porosité corticale du radius. De plus, l'imagerie a révélé également des altérations microarchitecturales (diminution du volume osseux et de l'épaisseur des travées osseuses) et une réduction de la force de l'os au niveau du tibia. Les auteurs ont établi un lien intéressant avec le contrôle glycémique ; les filles qui présentaient un pauvre contrôle glycémique, défini par une valeur d'hémoglobine glyquée supérieure à $8,5 \%$, démontraient des altérations microarchitecturales osseuses plus marquées. En conclusion, l'étude auprès d'une cohorte d'adolescentes atteintes de diabète de type 1 a mis en lumière une association entre l'hyperglycémie et des altérations microarchitecturales osseuses. Précédant les manifestations microvasculaires, ces altérations pourraient contribuer à la fragilité squelettique prévalente chez les personnes diabétiques.

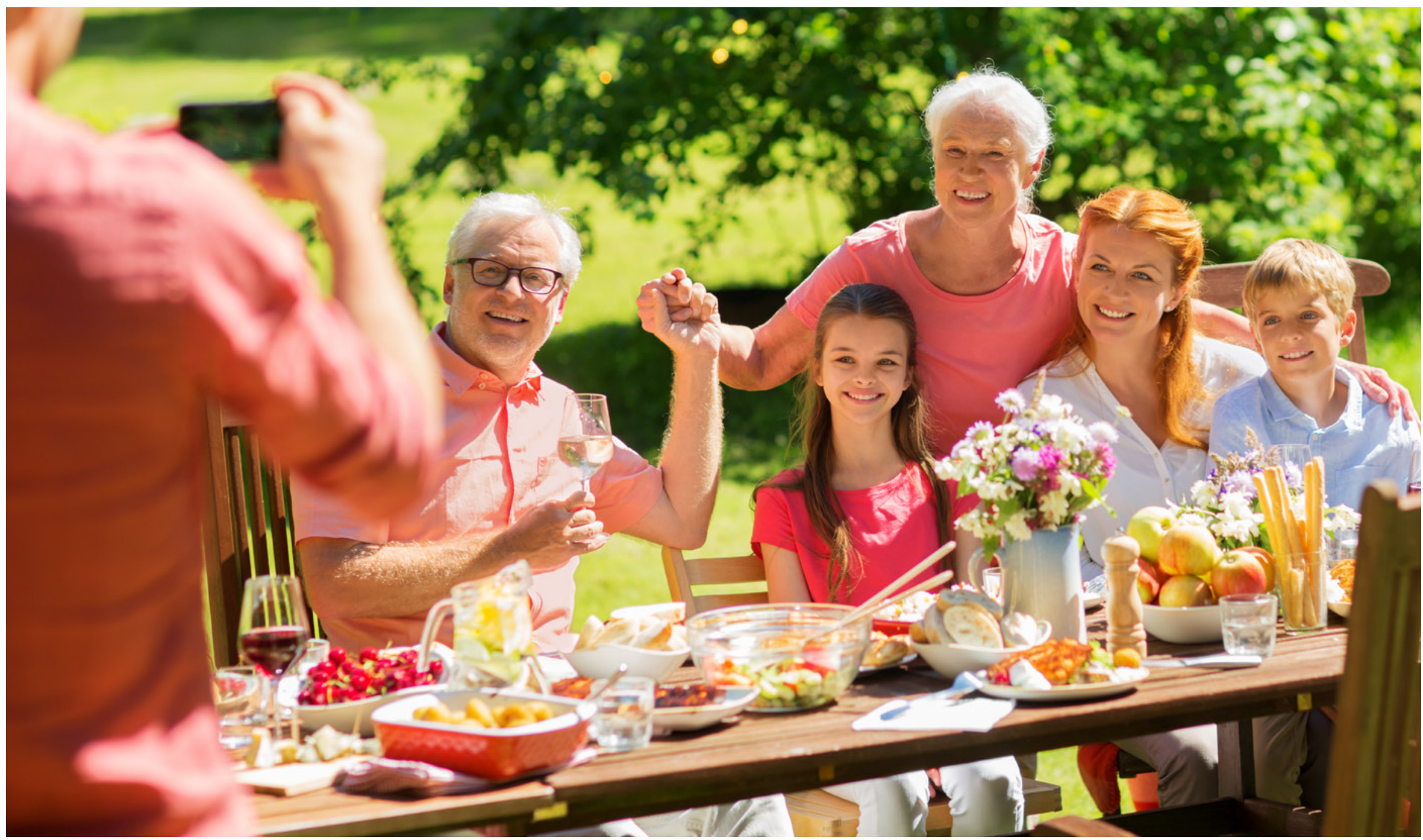

